\title{
BMJ Open Anti-inflammatory effect of prophylactic macrolides on children with chronic lung disease: a protocol for a double- blinded randomised controlled trial
}

\author{
Ricardo A Mosquera, ${ }^{1}$ Ana M Gomez-Rubio, ${ }^{1}$ Tomika Harris, ${ }^{1}$ Aravind Yadav, ${ }^{1}$ \\ Katrina McBeth, ${ }^{1}$ Traci Gonzales, ${ }^{1}$ Cindy Jon, ${ }^{1}$ James Stark, ${ }^{1}$ Elenir Avritscher, ${ }^{1}$ \\ Claudia Pedroza, ${ }^{1}$ Keely Smith, ${ }^{1}$ Giuseppe Colasurdo, ${ }^{1}$ Susan Wootton, ${ }^{1}$ \\ Pedro Piedra, ${ }^{2}$ Jon E Tyson, ${ }^{1}$ Cheryl Samuels ${ }^{1}$
}

To cite: Mosquera RA, Gomez-Rubio AM, Harris T, et al. Anti-inflammatory effect of prophylactic macrolides on children with chronic lung disease: a protocol for a double-blinded randomised controlled trial. BMJ Open 2016;6:e012060 doi:10.1136/bmjopen-2016012060

- Prepublication history and additional material is available. To view please visit the journal (http://dx.doi.org/ 10.1136/bmjopen-2016012060).

Received 26 March 2016 Revised 14 July 2016 Accepted 25 August 2016

\section{(a) CrossMark}

${ }^{1}$ Department of Pediatrics, McGovern Medical School, University of Texas Health Science Center at Houston, Houston, Texas, USA

${ }^{2}$ Department of Virology and Microbiology, Baylor College of Medicine, Houston,

Texas, USA

Correspondence to Dr Ricardo A Mosquera; Ricardo.A.Mosquera@uth. tmc.edu

\section{ABSTRACT}

Introduction: Recent studies suggest that the high mortality rate of respiratory viral infections is a result of an overactive neutrophilic inflammatory response. Macrolides have anti-inflammatory properties, including the ability to downregulate the inflammatory cascade, attenuate excessive cytokine production in viral infections, and may reduce virus-related exacerbations. In this study, we will test the hypothesis that prophylactic macrolides will reduce the severity of respiratory viral illness in children with chronic lung disease by preventing the full activation of the inflammatory cascade.

Methods and analysis: A randomised double-blind placebo-controlled trial that will enrol 92 children to receive either azithromycin or placebo for a period of 3-6 months during two respiratory syncytial virus (RSV) seasons (2015-2016 and 2016-2017). We expect a reduction of at least $20 \%$ in the total number of days of unscheduled face-to-face encounters in the treatment group as compared with placebo group. Standard frequentist and Bayesian analyses will be performed using an intent-to-treat approach.

Discussion: We predict that the prophylactic use of azithromycin will reduce the morbidity associated with respiratory viral infections during the winter season in patients with chronic lung disease as evidenced by a reduction in the total number of days with unscheduled face-to-face provider encounters.

Ethics and dissemination: This research study was approved by the Institutional Review Board of the University of Texas Health Science Center in Houston on 9 October 2014. On completion, the results will be published.

Trial registration number: NCT02544984.

\section{INTRODUCTION}

For the past 3 years, the High Risk Children's Clinic (HRCC) at the University of Texas Health Science Center at Houston
(UTHealth) has been providing a medical home for medically complex children. We have demonstrated major benefits of fewer hospital admissions and emergency room (ER) visits while providing healthcare savings for high-risk chronically ill children including patients with chronic lung disease (CLD). ${ }^{1}$ These benefits have not been previously shown for medical homes for patients of any kind or age. ${ }^{2}{ }^{3}$ These benefits result primarily from $24 / 7$ access by phone to healthcare providers. The clinic offers same day appointments and provides coordination of care for this population. We now aim to further cut morbidity rates by developing specific outpatient interventions to augment the care for each major disorder that we treat.

Almost half $(44 \%)$ of our patients in the HRCG are chronically ill children who have some form of CLD including patients with bronchopulmonary dysplasia (BPD). ${ }^{1}$ CLD, as defined by the American Thoracic Society (ATS) statement from 2002, is 'a heterogeneous group of respiratory diseases of infancy that usually evolves from an acute respiratory disorder experienced by a newborn infant ${ }^{4}$ specifically, infants with BPD-defined as the need for supplemental oxygen therapy in children over 28 days old that were born before 32 weeks gestation. ${ }^{4}$ These infants often incur long-term pulmonary function abnormalities including oxygen dependency after discharge, recurrent respiratory infections and other reactive airway diseases. From our data, we have learnt that many of the hospital admissions in our group of patients were related to respiratory infections $(37 \%)$ during the winter season. Despite vaccination rates of nearly $100 \%$, administration of palivizumab 
to all of those eligible patients and access to our comprehensive care clinic, viral respiratory illnesses continue to cause considerable morbidity and high healthcare costs in this patient population. ${ }^{1}$ Innovative new prophylactic treatments are needed.

Macrolides have received considerable attention for their anti-inflammatory and immunomodulatory actions. Such properties may ensure some efficacy against a wide spectrum of respiratory viral infections. ${ }^{5}$ Recent studies, including a study performed in our laboratory with elderly BALB/c mice infected with respiratory syncytial virus (RSV), have shown that the high mortality rate of respiratory virus infections is a result of an overactive neutrophilic inflammatory response. ${ }^{6} 7$ A recently published study examined the inflammatory response in hospitalised infants with RSV and evaluated the predictive value of cytokines in nasopharyngeal aspirate in comparison to disease severity and found an increase in Th1 and Th2 cytokines. ${ }^{8}$ Respiratory viral infections are characterised by the appearance of cytokine storms which involve an extreme production and secretion of numerous proinflammatory cytokines. Severity of infection is closely related to virus-induced cytokine dysregulation, which is responsible for the development of fatal clinical symptoms, such as massive pulmonary oedema, acute bronchopneumonia, alveolar haemorrhage and acute respiratory distress syndrome. ${ }^{5}$ Macrolides downregulate the inflammatory cascade, attenuate excessive cytokine production in viral infections and may reduce virusrelated exacerbation. ${ }^{5}$

Clinical trials have demonstrated controversial results in the effects of macrolides in respiratory viral infections. $^{9-11}$ To date, studies have only evaluated macrolide use as a treatment, not as a prophylactic therapy. Long-term therapy with the macrolide antibiotic erythromycin was shown to alter the clinical course of diffuse pan bronchiolitis in the late 1980s. ${ }^{12}$ Since then, macrolides have been found to have a large number of anti-inflammatory properties in addition to their antimicrobial effect. These observations provided the rationale for many studies performed over the last decade to assess the usefulness of macrolides in other inflammatory airways diseases including cystic fibrosis, asthma, chronic obstructive pulmonary disease (COPD) and bronchiolitis obliterans syndrome. $^{13}$ One randomised controlled trial (RCT) looked at the daily use of macrolides for up to 6 weeks to prevent BPD in premature infants in a neonatal intensive care unit (NICU) setting and found the neonates had better outcomes without an increase in adverse effects. ${ }^{14}$ However, the chronic use of macrolides to prevent respiratory infection complications in patients with CLD of infancy has not been studied. We will test the hypothesis that prophylactic macrolides are effective in reducing the severity of respiratory viral illness by preventing the full activation of an inflammatory cascade.

\section{METHODS AND ANALYSIS}

\section{Study design}

A single-site double-blinded RCT that will enrol 92 children age 6 months to 6 years who have CLD secondary to BPD during two respiratory viral seasons defined as 1 October to 31 March of each year (2015-2016 season and 2016-2017 season). At the conclusion of the first season, an interim analysis will be performed to justify the need for the second season. Clinic electronic healthcare records will be screened to determine eligibility.

\section{Study intervention}

This will be a pragmatic study design with a rolling enrolment time period from 1 October until 31 December. At enrolment, patients who have parental consent will undergo a baseline ECG, a nasal aspirate and an oscillometer reading (over 2 years of age only). At the initial study visit (which will be done during a regularly scheduled follow-up clinic visit), enrolees will receive a 6-month supply of either the medication or the placebo. All patients will take the medication until the end of the treatment phase (31 March). Patients will therefore end up receiving the medication for a time period ranging from 3 to 6 months, and extra medication will be discarded. The medication or placebo will be taken once a day 3 days a week (Monday, Wednesday and Friday). The azithromycin medication will be dosed at $5 \mathrm{mg} / \mathrm{kg} /$ day. Any child who is eligible to receive palivizumab will receive it every 28-30 days in clinic as per usual care. Patients will be monitored closely for adverse reactions over phone, in clinic during their regularly scheduled appointments, and/or during any necessary illness visits. Any children with adverse reactions will discontinue the medication but will continue to be followed clinically. At any clinic visit in which a child presents with respiratory infections including pneumonia, upper respiratory illness, bronchiolitis, etc, he/she will have an additional nasal aspirate and/or tracheal aspirate (if applicable) and an oscillometer reading (only for children $>2$ years) performed. At the completion of the treatment phase, each child will have a final nasal aspirate and/or tracheal aspirate, and an oscillometer reading performed. Data will continue to be collected for the following 2 months (1 April to 31 May), to monitor for respiratory illnesses and possible side effects.

\section{Study population}

High risk children, born before 37 weeks gestation with a current diagnosis of CLD secondary to BPD between the chronological age of 6 months and $<6$ years, who attend either the HRCG or the High Risk Infant Clinic (HRIC) at UTHealth at the McGovern Medical School in Houston, Texas USA, will be screened by the clinic providers. The HRIC follows premature infants born before 32 weeks gestation for their first 2 years of life; the high risk paediatric clinic follows medically complex 
children who have had at least three emergency department (ED) visits, two hospitalisations, and/or one paediatric intensive care unit (PICU) visit within the last year for a chronic health condition. We have chosen to exclusively recruit from these two clinics because $\sim 90 \%$ of premature children with CLD from the UTHealth System are followed up at either one of these two clinics. We will ensure patient recruitment by screening both clinics weekly during the enrolment process. Eligible patients will be approached by the research coordinator and/or one of the providers. Patients who have further questions will be given time to think and will be approached later with a phone call or next time they are in clinic.

\section{Inclusion/exclusion criteria}

All children who currently attend either the HRCC or the HRIC who are between 6 months and 6 years at the time of enrolment and meet the ATS definition of CLD secondary to BPD will be screened. CLD, as defined by the ATS statement from 2002, is 'a heterogeneous group of respiratory diseases of infancy that usually evolves from an acute respiratory disorder experienced by a newborn infant'. ${ }^{4}$ BPD is defined as (1) infants born $<32$ weeks who need supplemental oxygen for at least 28 days or (2) infants born between 32 weeks and 36 weeks who need supplemental oxygen for at least 56 days. ${ }^{4}$ All screening will be done by clinic providers (table 1).

Exclusion criteria include children with cystic fibrosis or bronchiectasis, ${ }^{15}$ because the prophylactic use of macrolides has already demonstrated value and become usual care for these patients. Children with cardiac arrhythmias will be excluded due to the potential increase in cardiovascular death that has been shown in the adult population. ${ }^{16}$ Patients with known cyanotic heart disease will be excluded. Children with colitis or short bowel syndrome will also be excluded due to the potential effects to the gastrointestinal flora or

\begin{tabular}{ll} 
Table 1 Inclusion and exclusion criteria \\
\hline Inclusion criteria & Exclusion criteria \\
\hline $\begin{array}{l}\text { Age of } 6 \text { months to 6 years } \\
\text { during respiratory viral }\end{array}$ & Cystic fibrosis or \\
season (1 October-31 & bronchiectasis \\
December) & Cardiac arrhythmias \\
Diagnosis of chronic lung & Cyanotic heart \\
disease (CLD) secondary to & disease \\
bronchopulmonary dysplasia & Colitis \\
(BPD) as defined by the & Known macrolide \\
American Thoracic Society & allergy \\
(ATS). & Taking medications \\
Receive primary care at & known to interact with \\
High Risk Infant Clinic & macrolides \\
(HRIC) or High Risk & Short bowel \\
Children's Clinic (HRCC) & Syndrome \\
\hline
\end{tabular}

malabsorption. In addition, any child with a known macrolide allergy or who is taking any medication that has a known interaction with macrolides and any child with kidney or liver failure will also be excluded.

\section{Study procedures}

After patients are screened as eligible, they will be approached during a routine office visit in the clinic. If the patient is interested in participating in the study, a baseline ECG will be conducted to ensure that patients enrolled do not have a prolonged QT interval or any other undiagnosed arrhythmias. If the ECG is normal, written informed consent will be obtained in the clinic from the parent or legal guardian of each eligible child by any of the coinvestigators or the research nurse at the time of enrolment.

Once a patient is deemed eligible, he or she will be randomised to either azithromycin or placebo using the REDCap randomisation module. Allocation ratio will be 1:1 and will be stratified by use of palivizumab and presence of tracheostomy. The statistician will create the randomisation sequence using labelling of $\mathrm{A}$ or $\mathrm{B}$ for the two groups and will upload it into REDCap. This will be double-blinded as neither the providers nor the patients will know whether they are receiving placebo or medication. Allocation concealment will be ensured, as the allocation sequence is only known to the study statistician (who is also blinded to the labelling of the groups) and is not made available in REDCap until after the patient has been recruited into the trial.

After randomisation, the research nurse or another clinical member of the HRCC team will then collect a nasal aspirate sample at the first study visit (description in Laboratory section). The nasal aspirate will be stored and studied after the conclusion of the treatment phase for its levels of myeloperoxidase (MPO), cytokines, respiratory virology and microbiome. In addition, all patients two and older who can, will have a spirometry reading performed using a TremoFlo airway oscillometry system (AOS) manufactured by Thorasys. Patients will be recruited and enrolled for this study on a rolling basis from 1 October to 31 December. Once enrolled, all participants will complete the intervention phase of the protocol on 31 March. Half of the patients will receive azithromycin at a dose of $5 \mathrm{mg} / \mathrm{kg}$ to be given once a day on Monday, Wednesday and Friday. The other half, the control group, will be provided with a placebo medication of similar taste, colour, texture and consistency, also to be taken once a day on Monday, Wednesday and Friday. The study medication and the placebo will have a fish oil base to ensure a shelf life of $>6$ months and flavoured with citrus to improve palatability. Parents will be contacted monthly, either in clinic or by phone, to monitor for their progress and potential adverse reactions. If a significant adverse reaction occurs, the medication will be discontinued. If an allergic reaction (such as rash or shortness of breath) is noted, the blind will be broken by the statistician, who is not involved with 
patient care or data collection. This unblinding will be done to note if it is an allergy to the azithromycin or the preparation. Confidentiality will be maintained as patients will be identified using only a study identification number. All data will be entered into a Health Insurance Portability and Accountability Act of 1996 (HIPAA) compliant database, and all paper copies will be kept in a locked office to ensure patient confidentiality.

After the initial appointment, at any face-to-face encounter (unscheduled sick visit or hospital or ED admission Monday through Friday) in which the patient presents with respiratory symptoms, the patient will be evaluated by the research nurse or one of the coinvestigators. Specifically, if a patient presents with the following symptoms: cough, wheeze, tachypnoea, rhinorrhoea, increased respiratory secretions, hypoxaemia, and/or an increased oxygen requirement, an additional nasal aspirate sample and, if applicable, a tracheal aspirate will be done. Oscillometer reading will also be performed for those above 2 years of age when the patients are in clinic during each sick clinic visit for respiratory illness or after the study. At the conclusion of the 3-6 months treatment phase, a final nasal aspirate and/or tracheal aspirate and an ECG will be collected. Additionally, an oscillometer reading will also be collected in those patient 2 years of age and older. There will be no expected/additional study visits and no compensation will be provided for parents or patients.

\section{Risks}

Potential risks include an allergic reaction or adverse reaction to the medication or placebo. Examples of potential side effects include nausea, vomiting, diarrhoea and skin reactions. In addition, there may be an increased risk of diaper rash and/or oral thrush with the increased use of antibiotics. Other adverse outcomes associated with azithromycin include an increase in pyloric stenosis with prenatal and infant exposure for the first 4 months of life; ${ }^{17}$ however, our population for this project will be $>6$ months of age as approved by the Institutional Review Board (IRB). Any changes to the protocol or staff will be communicated by the research coordinator to the UTHealth IRB using the Integrated Research Information System (iRIS). Each of these risks and any other unexpected outcomes will be monitored at any visits to the clinic, or with monthly phone calls to see if any patients experience any adverse events. If there is a symptom, the providers will be made aware, and if in their professional opinion they believe the event is an adverse reaction to the medication, it will be recorded in the REDCap database, and parents will be advised to stop the medication immediately. In case of any serious adverse events, the IRB will also be notified and, if needed, the statistician will break the blind. With the anti-inflammatory properties of the macrolide, we predict an overall reduction in the severity of respiratory illnesses during the study period. Additionally, we will continue to monitor patients for a 2-month period following the last azithromycin administration as the medication may lead to a more lasting reduction in the number of unscheduled office visits, ER visits and hospital admissions. With less face-to-face provider encounters, there will be less opportunity for potential exposure to other viruses, as well as less time away from home or work for the patients' parents and, potentially, decreased healthcare-related costs.

\section{Study outcomes}

Our primary outcome is the total number of days of unscheduled face-to-face encounters for all diagnoses (defined as unscheduled sick visits, urgent care visits, ER visits and hospital admissions) during the 3-6 month treatment phase of the study.

\section{Secondary outcomes}

1. Individual component of the primary outcomeunscheduled sick visits, urgent care visits, ER visits and hospital admissions

2. Adverse side effects including gastrointestinal upset (vomiting/diarrhoea) and diaper rash

3. Total hospital and clinic costs from a healthcare system perspective. We expect the intervention to be cost-effective from a healthcare system perspective defined as following:
A. Decreased days of care without increasing cost
B. Decreased cost without increasing days of care
C. Decreased days of care while decreasing cost

4. Level of MPO in the nasopharyngeal secretions collected during respiratory illnesses that require a face-to-face provider encounter

5. Level of proinflammatory cytokines in the nasopharyngeal secretions collected during respiratory illnesses that require a face-to-face provider encounter

6. Level of airway obstruction as measured by a TremoFlo AOS during the 3-6 month treatment phase during respiratory illness and at the conclusion of the intervention phase

\section{Laboratory evaluation}

Microbiologic studies for exploratory outcomes will be conducted. The study involves collecting a minimum of two nasal aspirates from each patient. Nasal aspirate samples will be tested to measure the levels of MPO and lactate dehydrogenase (LDH); we will also use ELISA tests to measure the levels of specific proinflammatory cytokines, including interleukin 8 (IL-8) and interleukin 6 (IL-6), and PCR, in order to screen for several major respiratory viral pathogens.

Nasal aspirate specimens will be collected at the first visit, the final visit, and during any episodic respiratory sick visits at clinic or at Children's Memorial Hermann Hospital during regular office hours. Once collected, the specimen will be immediately diluted (1:1 solution) with viral transport medium (15\% glycerol in Iscove's media). The final solution (aspirated specimen mixed 
with viral transport medium) will contain a maximum of $9 \mathrm{~mL}$ (with a range of 3-9 mL). After dilution, the specimens will be immediately refrigerated at $37^{\circ}$ Fahrenheit until transportation to the freezers, where they will be stored at $-80^{\circ} \mathrm{C}$. The samples will be frozen and stored until the completion of the trial (table 2).

\section{Laboratory procedures}

The level of MPO will be measured using reagent tests, and several cytokines that are markers of inflammation will be tested using ELISA tests; details of the primers and probes to be used are found in a 2005 article by Beckham $e t a l^{18}$ In addition, reverse transcription PCR (RT-PCR) assays will be performed using TaqMan-based primers and probes to detect the presence of 5-6 major respiratory viruses, and the remaining volume of aspirate will be stored for future studies of additional biomarkers. Nasal aspirate samples will be collected for assays of up to four cytokines and chemokines. Samples will be tested according to the manufacturer's instructions. Samples and serial dilutions of the cytokine standards will be incubated with antihuman cytokine-coated beads in a 96-well filtration plate.

\section{Data collection, management and analysis plan}

All data will be kept in REDCap database. REDCap is a HIPAA compliant and safe database that can be accessed from any computer with an internet access. It is backed up on a regular basis. Collected data at baselines include: demographic information, baseline ECG, baseline nasal aspirate and baseline oscillometer reading. At the conclusion of the study, the ECG and nasal aspirate will be repeated. If any patient presents to the clinic, ED or hospital with respiratory symptoms during business hours, a nasal aspirate and oscillometer reading will be collected. Data on when symptoms started and the symptoms experienced will also be collected.

Data can only be entered into REDCap by the research coordinator. At the end of the medication period, the research coordinator will ensure that all encounters were entered correctly by double checking the medical records. Each patient will be called once a month to follow-up on their medication status and to assess for any possible side effects.

Standard frequentist and Bayesian analyses will be performed using an intent-to-treat approach. Total number of days with unscheduled face-to-face encounters, total hospital admissions (counting each hospitalisation as an event), total ER/urgent care visits (counting one day for each ER/urgent care visit), and total unscheduled clinic visits (counting one day for each visit) will be analysed and related to treatment group (azithromycin vs placebo) using Poisson regression models with robust SEs to estimate relative risks (RRs) and 95\% CIs. All models will include treatment group and stratification variables (use of palivizumab and current tracheostomy) as covariates and length of follow-up as an offset. To assess the probability of benefit, we will use Bayesian models with interaction terms between treatment group (azithromycin vs placebo) and two predefined potential moderators-use of palivizumab and current tracheostomy. The conservative Bayesian approach of Dixon and Simon allows us to shrink the subgroup estimates to the overall mean treatment effect. For all Bayesian analyses, prior distributions for all regression coefficients will be centred at RR of 1.0 (normal with mean 0 and SD of 1 in the $\log$ scale), and half-normal $(0,1)$ for SD parameters. Point estimates of treatment effect and 95\%

Table 2 Study schedule

\begin{tabular}{|c|c|c|c|c|}
\hline \multirow[b]{2}{*}{ Time point } & \multicolumn{4}{|l|}{ Study period } \\
\hline & $\begin{array}{l}\text { Enrolment } \\
\text { October-December }\end{array}$ & $\begin{array}{l}\text { Allocation } \\
\text { October-December }\end{array}$ & $\begin{array}{l}\text { Post allocation } \\
\text { October-March }\end{array}$ & $\begin{array}{l}\text { Close out } \\
\text { March-October }\end{array}$ \\
\hline \multicolumn{5}{|l|}{ Enrolment } \\
\hline Eligibility screen & $\mathrm{x}$ & & & \\
\hline Informed consent & $\mathrm{x}$ & & & \\
\hline ECG & $\mathrm{x}$ & & & \\
\hline Nasal aspirate & $\mathrm{x}$ & & $\mathrm{x}$ & \\
\hline Oscillometer & $\mathrm{x}$ & & & \\
\hline Allocation & & $x$ & & \\
\hline \multicolumn{5}{|l|}{ Intervention } \\
\hline Take medicine & & & $\mathrm{x}$ & \\
\hline Take placebo & & & $\mathrm{x}$ & \\
\hline \multicolumn{5}{|l|}{ Assessment } \\
\hline Demographics & $\mathrm{x}$ & & & \\
\hline Unscheduled clinic visits & & & & $\mathrm{x}$ \\
\hline Unscheduled hospital visits & & & & $\mathrm{x}$ \\
\hline Emergency room visits & & & & $\mathrm{x}$ \\
\hline Evaluation of nasal aspirates & & & & $\mathrm{x}$ \\
\hline Analysis & & & & $\mathrm{x}$ \\
\hline Publication & & & & $\mathrm{x}$ \\
\hline
\end{tabular}


credible intervals will be reported along with probability of treatment benefit.

\section{Sample size and power}

Based on data from our HRCC, we expect the placebo group to have 1.6 encounters per child-year $(\mathrm{SD}=1.66)$. Assuming a two-sided $\alpha$ of 0.05 , a sample size of 92 (46/ group) will have $80 \%$ power to detect a difference of 1 in the encounter rate between placebo and azithromycin groups (ie, 1.6 vs 0.6 in encounter rate or $38 \%$ reduction). Power will be more limited for secondary outcomes, but Bayesian analyses will provide an estimate of the probability of benefit in these outcomes. A reduction of 1 encounter per child-year in the HRCC and the HRIC is clinically significant and realistically achievable. Since our clinics have been proven to decrease the number of days of care given outside the home by providing comprehensive care, we believe that the reduction of face-to-face provider encounters could be more pronounced in usual care.

\section{Ethics and dissemination}

This research study was approved by the IRB of the UTHealth in Houston on 9 October 2014 (HSC-MS-14-0476) (see online supplementary appendix A). Parental informed consent was obtained at time of enrolment by either the provider who approached the family or the research coordinator. Details concerning the enrolment process can be found in the Standard Protocol Items: Recommendations for Interventional Trials (SPIRIT) checklist (see online supplementary appendix $\mathrm{B})$.

Results from this trial will be published on completion in a peer reviewed scientific journal.

\section{DISCUSSION}

A substantial portion of our high risk chronically ill children have some form of CLD, including patients with tracheostomies, BPD, and chronic respiratory failure requiring mechanical ventilation. Most of the hospital admissions in this group of patients were related to respiratory infections in children $<6$ years of age $(37 \%$ during the winter season for the previous 3 years). Despite vaccination rates of nearly $100 \%$ and $24 / 7$ access to our clinic, viral respiratory illnesses continue to cause considerable morbidity and high healthcare costs. ${ }^{1}$ Innovative new prophylactic treatments are needed.

With this proposal, we will determine if the prophylactic use of azithromycin will (1) reduce the total number of days when unscheduled medical treatment healthcare-related encounters was given outside the home, (2) reduce the number of ER/urgent care visits, hospitalisations and clinic visits due to respiratory illness, (3) reduce the level of MPO and proinflammatory cytokines during viral illnesses requiring face-to-face physician interaction, (4) demonstrate a reduction in airway obstruction as measured by an oscillometer (5) have a similar safe profile compared with the placebo and (6) demonstrate cost-effectiveness of macrolides use. Understanding the anti-inflammatory effects of azithromycin when used as a prophylactic drug will provide important insight into the prevention of more serious sequelae of respiratory infections. In particular, this study will contribute to understanding disease in children ages 6 months to 6 years with CLD, a population that has a higher rate of hospitalisations for respiratory symptoms.

We predict that the prophylactic use of azithromycin will reduce the morbidity associated with respiratory viral infections during the winter season in patients with CLD as evidenced by a reduction in the days with unscheduled face-to-face provider encounters based on the preliminary results from our laboratory study that indicated that prophylactic azithromycin can effectively reduce airway inflammation and disease severity in a RSV-infected mouse model. ${ }^{6}{ }^{7}$ Recent studies have shown that the high morbidity rate of respiratory virus infections is a result of a neutrophilic overactive inflammatory response. ${ }^{5}$ Macrolides downregulate the inflammatory cascade, attenuate excessive cytokine production in viral infections and may reduce virus-related exacerbation. $^{15}$

Contributors RAM, GC, CJ, KS, JS, AY, KMcB, TG, EA, CP, CS, TH, AMG-R, $S W, P P$ and JET were involved in the concept and design of the study. RAM, $\mathrm{CJ}, \mathrm{AY}, \mathrm{KMcB}, \mathrm{TG}, \mathrm{CS}, \mathrm{TH}$ and $\mathrm{AMG}-\mathrm{R}$ were involved in enrolment. CS, TH and AMG-R were involved in daily research activities. RAM, JS, EA, CP, CS, AMG-R, SW, PP and JT were involved in the manuscript preparation and approval of the final manuscript. RAM wrote the initial protocol. AMG-R helped with the editing of tables and figures and the refining of the protocol. JS helped in editing the protocol and gave suggestions to improve the methodology of the protocol. CP was involved in writing the statistical analysis section of methods. SW and PP were involved in the planning and writing of the microbiology/laboratory portion of the protocol. JT mentored and adviser the primary investigator and was involved in the revision of the protocol. CS helped create, write, finalise, edit and refine the protocol from the rough draft to the final protocol.

Funding This research received no specific grant from any funding agency in the public, commercial or not-for-profit sectors.

Competing interests None declared.

Ethics approval University of Texas Health Science Center Committee for the protection of human subjects.

Provenance and peer review Not commissioned; externally peer reviewed.

Open Access This is an Open Access article distributed in accordance with the Creative Commons Attribution Non Commercial (CC BY-NC 4.0) license, which permits others to distribute, remix, adapt, build upon this work noncommercially, and license their derivative works on different terms, provided the original work is properly cited and the use is non-commercial. See: http:// creativecommons.org/licenses/by-nc/4.0/

\section{REFERENCES}

1. Mosquera RA, Avritscher EB, Samuels CL, et al. Effect of an enhanced medical home on serious illness and cost of care among high-risk children with chronic illness: a randomized clinical trial. JAMA 2014;312:2640-8. doi:10.1001/jama.2014.16419.

2. Homer CJ, Klatka K, Romm D, et al. A review of the evidence for the medical home for children with special health care needs. Pediatrics 2008;122:e922-937. 
3. Jackson GL, Powers BJ, Chatterjee R, et al. The patient centered medical home: a systematic review. Ann Intern Med 2013;158:169-78.

4. Allen J, Zwerdling R, Ehrenkranz R, et al., American Thoracic Society. Statement on the care of the child with chronic lung disease of infancy and childhood 2003;168:356-96.

5. Min JY, Jang YJ. Macrolide therapy in respiratory viral infections. Mediators Inflamm 2012;2012:649570.

6. Mosquera RA, Stark JM, Atkins CL, et al. Functional and immune response to respiratory syncytial virus infection in aged BALB/C mice: a search for genes determining disease severity. Exp Lung Res 2013;40:40-9.

7. Mosquera R. Prophylactic azithromycin reduces airway inflammation and mortality in a mouse model of RSV infection. Denver, CO: American Thoracic Society, 2015.

8. Moreno-Solís G, Torres-Borrego J, de la Torre-Aguilar MJ, et al. Analysis of the local and systemic inflammatory response in hospitalized infants with respiratory syncitial virus bronchiolitis. Allergol Immunopathol (Madr) 2015;43:264-71.

9. Pinto LA, Pitrez PM, Luisi F, et al. Azithromycin therapy in hospitalized infants with acute bronchiolitis is not associated with better clinical outcomes: a randomized, double-blinded, and placebo-controlled clinical trial. J Pediatr 2012;161:1104-8.

10. Kneyber MC, van Woensel JB, Uijtendaal E, et al. Azithromycin does not improve disease course in hospitalized infants with respiratory syncytial virus (RSV) lower respiratory tract disease: a randomized equivalence trial. Pediatr Pulmonol 2008;43:142-9.

11. Tahan F, Ozcan A, Koc N. Clarithromycin in the treatment of RSV bronchiolitis: a double-blind, randomised, placebo-controlled trial. Eur Respir J 2007;29:91-7.

12. Friedlander AL, Albert RK. Chronic macrolide therapy in inflammatory airways diseases. Chest 2010;138:1202-12.

13. Cai $Y$, Chai $D$, Wang R, et al. Effectiveness and safety of macrolides in cystic fibrosis patients: a meta-analysis and systematic review. $J$ Antimicrob Chemother 2011;66:968-78.

14. Ballard HO, Anstead MI, Shook LA. Azithromycin in the extremely low birth weight infant for the prevention of Bronchopulmonary Dysplasia: a pilot study. Bio Med Central. 2007;8:41.

15. Corvol $\mathrm{H}$, Taytard J, Thouvenin G, et al. [Why use long-term macrolide therapy in pediatric pulmonology?]. Arch Pediatr 2014;21:314-21.

16. Ray WA, Murray KT, Hall K, et al. Azithromycin and the risk of cardiovascular death. N Engl J Med 17 2012;366:1881-90.

17. Lund M, Pasternak B, Davidsen RB, et al. Use of macrolides in mother and child and risk of infantile hypertrophic pyloric stenosis: nationwide cohort study. BMJ 2014;348:g1908.

18. Beckham JD, Cadena A, Lin J, et al. Respiratory viral infections in patients with chronic, obstructive pulmonary disease. $J$ Infect 2005;50:322-30. 